\title{
Management of Anesthesia in Adult and Pediatric Mastocytosis: A Study of the Spanish Network on Mastocytosis (REMA) Based on 726 Anesthetic Procedures
}

\author{
Almudena Matito ${ }^{a, b}$ José Mario Morgado a, b Paula Sánchez-López ${ }^{b, c}$ \\ Iván Álvarez-Twose ${ }^{a, b}$ Laura Sánchez-Muñoz ${ }^{a, b} \quad$ Alberto Orfao $^{b, d}$, e \\ Luis Escribano ${ }^{\text {b, d }}$

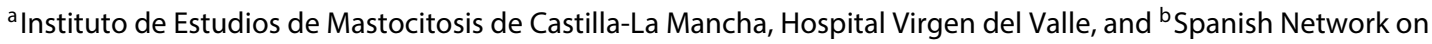 \\ Mastocytosis (REMA), Toledo, CAllergy Department, Hospital Rey Juan Carlos, Móstoles, and d Servicio General de \\ Citometría, Centro de Investigación del Cáncer/IBMCC (USAL/CSIC), and e Departamento de Medicina, Universidad \\ de Salamanca and IBSAL, Salamanca, Spain
}

\section{Key Words}

Anesthesia - Anaphylaxis - Mast cell mediator

release-associated symptoms · Mastocytosis

\begin{abstract}
Background: The role of anesthesia as an elicitor of mast cell (MC) mediator release symptoms in mastocytosis is poorly investigated. Objective: To determine the frequency and type of MC mediator release symptoms during anesthetic procedures in mastocytosis patients. Methods: Medical records were reviewed regarding the anesthetic techniques for 501 mastocytosis patients ( 459 adults and 42 children; 95 and $5 \%$ with systemic involvement, respectively) who were subjected to 676 and 50 anesthetic techniques, respectively. General, sedation, epidural, and local anesthetic techniques were used in $66(10 \%), 67(10 \%), 76(11 \%)$, and $515(76 \%)$ adult patients and in 24 (48\%), 8 (16\%), 2 (4\%), and $25(50 \%)$ pediatric patients. Results: The frequency of perioperative
\end{abstract}

MC mediator-related symptoms and anaphylaxis was 2 and $0.4 \%$ in the adult series and 4 and $2 \%$ among children. In the adult series, this frequency was significantly higher in patients who previously presented with anaphylaxis $(p=0.03)$, underwent major surgeries $(p<0.001)$ and general anesthesia $(p=0.02)$, and were not given prophylactic antimediator therapy (PAT) $1 \mathrm{~h}$ before the anesthesia $(\mathrm{H} 1 / \mathrm{H} 2$ antihistamines and benzodiacepines; $p=0.002$ ). Hypersensitivity and/or allergy to the involved drugs and latex allergy were ruled out in all but one symptomatic case; when PAT was given and sedation was added, some cases later tolerated the same anesthetic drugs. Conclusion: The frequency of perioperative anaphylaxis appears to be higher in mastocytosis patients than in the general population. Mastocytosis should not be a contraindication for anesthesia since PAT and adequate anesthetic management using the drugs with the safest profile appears to be effective in preventing/controlling MC mediator-associated symptoms.

C 2015 S. Karger AG, Basel

\section{KARGER 125}

(c) 2015 S. Karger AG, Base

$1018-2438 / 15 / 1671-0047 \$ 39.50 / 0$

E-Mail karger@karger.com

www.karger.com/iaa
Correspondence to: Dr. Almudena Matito

Instituto de Estudios de Mastocitosis de Castilla-La Mancha

Hospital Virgen del Valle

Carretera de Cobisa s/n, ES-45071 Toledo (Spain)

E-Mail amatito@sescam.jccm.es 


\section{Introduction}

Mastocytosis includes a heterogeneous group of disorders characterized by the presence of abnormal mast cells (MC) in different organs and tissues. Because of its heterogeneity, mastocytosis is classified into different categories based on its clinical presentation and the pattern and extent of tissue involvement. Thus, mastocytosis is classified according to the age of onset (pediatric vs. adult), the organs involved (cutaneous vs. systemic), and its clinical behavior/MC burden (indolent vs. aggressive disease). Indolent systemic mastocytosis (ISM) is the most common form of the disease, and it is typically associated with a good prognosis and a normal life expectancy in patients with the D816V KIT mutation restricted to the MC compartment $[1,2]$.

Patients with mastocytosis frequently present cutaneous and systemic symptoms due to the release of MC mediators, including pruritus, urticaria, flushing, gastrointestinal complaints (nausea, abdominal pain, and diarrhea), shortness of breath, and even anaphylaxis. Such symptoms may emerge spontaneously or can be triggered by several different factors. Anaphylaxis due to a massive MC mediator release is present in a significant portion of systemic mastocytosis (SM) patients $(22-49 \%)[3,4]$ and can be elicited by either known or unknown triggers through diverse mechanisms involving IgE- or non-IgEmediated pathways. Among other triggers, anesthesia may induce MC mediator release [5-7] associated with mechanical irritation of tissues, stress or drugs such as neuromuscular blockers, opioids, hypnotics, analgesics, and colloids [5, 6, 8-11]. Currently, there is limited data on the frequency of perianestethic MC mediator symptoms in patients with mastocytosis, and most available information is based on case reports. In the few (limited) series of pediatric mastocytosis patients $[10,12,13]$ and in the single large series of adult mastocytosis patients [14] who underwent anesthetic procedures reported thus far, the frequency of anaphylaxis was 0 and $0.6 \%$, respectively. Despite this, anesthetic procedures are considered high risk in mastocytosis since severe reactions (e.g. systemic hypotension/anaphylactic reactions and coagulopathy) resulting in death in individual patients have been recurrently reported [15-25]. Because of this, several different recommendations following single-center experiences have been proposed, including the use of prophylactic antimediator therapy (PAT) and some specific anesthetic drugs in order to prevent $\mathrm{MC}$ mediator release in anesthetic procedures in patients with mastocytosis $[10$, $13,16,20,26-36]$. Furthermore, determination of periop- erative serum tryptase is strongly recommended if MC mediator release is suspected to occur during an anesthetic procedure $[8,37,38]$.

Here, we report a retrospective study on a large series of 501 consecutive patients with mastocytosis ( 459 adults and 42 children) who underwent 726 anesthetic procedures; our aim was to determining the frequency and type of MC mediator episodes and symptoms associated with anesthesia and anesthetic procedures in distinct subtypes of mastocytosis.

\section{Materials and Methods}

\section{Patients}

The medical records of 501 consecutive patients ( 459 adults and 42 children) diagnosed with different categories of mastocytosis according to World Health Organization (WHO) criteria [39-42], who were followed by the Spanish Network on Mastocytosis (REMA) and underwent anesthetic procedures from 1989 to 2014, were reviewed. Every adult participant, as well as the children and their parents, gave their informed consent to participate in this retrospective study which was approved by the local ethics committee.

Of the 459 adults, 257 (56\%) were women and 202 (44\%) were men; of them, 13 (3\%) had cutaneous mastocytosis (CM), $9(2 \%)$ were classified as having mastocytosis in the skin (MIS) [in these cases a bone marrow (BM) study was not performed and systemic involvement was not ruled out], 259 (56\%) had indolent SM presenting with skin lesions $\left(\mathrm{ISMs}^{+}\right), 124(27 \%)$ had ISM without skin lesions (ISMs ${ }^{-}$), 27 (6\%) had well-differentiated SM (WDSM) [43], 20 (4\%) had aggressive SM, 4 (1\%) had MC leukemia, 1 had ISMs ${ }^{+}$ associated with another clonal hematological non-MC lineage disease (AHNMD) - i.e. non-Hodgkin B-cell lymphoma - and 2 had ISMs $^{-}$-AHNMD (a myeloproliferative/myelodisplastic syndrome in both patients). In the pediatric group, 19 (45\%) patients were girls and $23(55 \%)$ were boys, with the following diagnoses: cutaneous mastocytoma $(n=2 ; 5 \%)$, WDSM $(n=2 ; 5 \%), C M(n=4 ; 10 \%)$, and MIS ( $\mathrm{n}=34 ; 80 \%)$.

The onset of the disease was defined either by the moment in which the skin lesions appeared or by the time of the first anaphylaxis episode, or by the presence of B or C findings [40] in patients who presented without skin lesions. Adult patients had undergone between 1 and 7 anesthetic procedures for a total of 676 anesthetic procedures as follows: 1 procedure, $\mathrm{n}=333(72 \%) ; 2$ procedures, $\mathrm{n}=80(17 \%) ; 3$ procedures, $\mathrm{n}=21(5 \%) ; 4$ procedures, $\mathrm{n}=12(3 \%)$; 5 procedures, $\mathrm{n}=7(1 \%) ; 6$ procedures, $\mathrm{n}=5(1 \%)$, and 7 procedures, $\mathrm{n}=1(0.5 \%)$. Among the children, $36(86 \%), 4(9 \%)$, and 2 (5\%) patients had undergone 1, 2, and 3 anesthetic procedures, respectively, for a total of 50 anesthetic procedures. Every anesthetic procedure was classified according to the American Society of Anesthesiologists (ASA) physical status classification system [44], and every surgery was termed major or minor following previously reported criteria [45].

Data about MC mediator release-related symptoms (e.g. pruritus, flushing, gastrointestinal symptoms, and anaphylaxis) was specifically recorded for each anesthetic procedure with the specific antimediator therapy given for mastocytosis (e.g. oral disodium cromolyn, $\mathrm{H} 1$ and $\mathrm{H} 2$ antihistamines, leukotriene antagonists, and DOI: $10.1159 / 000436969$
Matito et al. 
corticosteroids), as well as the cytoreductive (interferon- $\alpha-2 b$, hydroxyurea, and cladribine) or targeted (imatinib) therapies administered during the year before the anesthetic procedure was carried out. Use of intensive antimediator therapy was defined based on administration of scheduled disodium cromolyn plus any of the above-mentioned antimediator drugs to control MC mediator-related symptoms. In addition, administration of $\mathrm{H} 1 / \mathrm{H} 2$ antihistamines, corticosteroids, montelukast, and/or benzodiacepines in different combinations before anesthesia was also considered. PAT was used when at least $\mathrm{H} 1$ antihistamines or corticosteroids had been administered before anesthesia. Finally, the occurrence of MC mediator-associated symptoms, excessive bleeding during the procedure or the perioperative period, the treatment required to control the symptoms, and the need for a blood transfusion after anesthesia were also systematically recorded in each case.

\section{Anesthetic Techniques}

Anesthetic procedures were classified according to the anesthetic techniques used as follows: general anesthesia, sedation, epidural anesthesia, or local anesthesia. In this line, every procedure could be performed using one or a combination of these techniques. From this point on, the number of anesthetic procedures will be referred to as cases.

Information about the specific anesthetic drugs used (e.g. inhalant anesthetics, neuromuscular blocking drugs, sedative and hypnotic drugs, local anesthetics, and opioids, as well as other drugs commonly used in anesthesia like neostigmine, atropine, nonsteroidal anti-inflammatory drugs, analgesics, antibiotics, and colloid fluids) was systematically recorded from the medical reports for each anesthetic procedure. Furthermore, the use of PAT before every risk procedure was also recorded.

Serum tryptase levels were determined using the available commercial technique (Phadia, Uppsala, Sweden/Thermo Fisher Scientific Inc.); in the majority of the patients, serum tryptase levels were determined in basal (clinical) conditions (sBT) during the year before the anesthesia took place.

\section{Allergological Work-Up}

In every perioperative symptomatic case, the suspected triggers (e.g. latex and drugs) were identified; subsequently, a complete allergic work-up was performed, including an evaluation of specific IgE levels when available (ImmunoCAP; Phadia/Thermo Fisher Scientific Inc.) and skin tests (skin prick tests and intradermal tests), as well as specific drug challenges.

\section{Statistical Methods}

The Mann-Whitney $U$ test or a $\chi^{2}$ test was used to assess the statistical significance of differences observed between groups for continuous and categorical variables, respectively. $p<0.05$ was considered statistically significant. For all statistical analyses, the IBM SPSS version 20 software package was used.

\section{Results}

The anesthetic techniques used for adult and pediatric mastocytosis cases included: general anesthesia in 66 $(10 \%)$ and $24(48 \%)$, sedation in $67(10 \%)$ and $8(16 \%)$, epidural in $76(11 \%)$ and $2(4 \%)$, and local anesthesia in $515(76 \%)$ and 25 (50\%) cases, respectively. The number of specific procedures carried out according to the anesthetic technique used is detailed in online supplementary table 1 (for all online suppl. material, see www.karger. com/doi/10.1159/000436969). Combination of 2 anesthetic techniques was carried out in 49 (7\%) adult and 9 (18\%) pediatric procedures, including sedation plus local anesthesia in $22(45 \%)$ adult and $3(6 \%)$ pediatric cases, sedation and epidural anesthesia in 17 (35\%) adult and 2 (4\%) pediatric cases, and general anesthesia plus either local or epidural anesthesia in $5(10 \%)$ adult and $4(8 \%)-$ local anesthesia - pediatric cases, respectively.

\section{Mediator-Related Symptoms during the \\ Perioperative Period}

Overall, 11 (2\%) adult cases and 2 (4\%) pediatric cases suffering from cutaneous mastocytoma $(\mathrm{n}=1$ child $), \mathrm{CM}$ ( $\mathrm{n}=1$ adult), MIS ( $\mathrm{n}=1$ child), and ISM ( $\mathrm{n}=10$ adults) who underwent general anesthesia ( $\mathrm{n}=4$ adults and 2 children), sedation ( $\mathrm{n}=2$ adults), and epidural ( $\mathrm{n}=2$ adults) and local ( $\mathrm{n}=4$ adults) anesthetic techniques had perioperative MC-related symptoms consisting of anaphylaxis in 3 of 11 adults ( $0.4 \%)$ and 1 of 2 children (2\%), respectively; the remaining cases presented mild symptoms. Of note, for 3 of 4 cases that presented perioperative anaphylaxis, this was the first anaphylaxis episode.

The detailed characteristics of the anesthetic procedures used in cases presenting perioperative MC-mediator release-associated episodes are shown in table 1. Anaphylaxis in adult cases presented as sudden hypotension in the absence of other perioperative causes, associated cardiorespiratory arrest and coagulopathy (case No. 2), generalized urticaria (case No. 6), and loss of consciousness (case No. 9); the pediatric case presented bronchospasm and generalized erythema. Unfortunately, tryptase or histamine levels were not determined during the anaphylactic period. Interestingly, when the medication used as PAT was given during a later anesthetic procedure to case No. 3 and 12, as well as when sedation was added in case No. 9, all 3 cases had tolerance to the anesthetic drugs previously involved as possible elicitors of symptoms. In all symptomatic cases, a tolerance to latex was demonstrated later on; in addition, hypersensitivity and/or allergy to the drugs involved was also systematically ruled out, except in case No. 10, in whom the allergological study was still pending at the time of reporting as he was still under antimediator therapy. In the majority of cases, the MC mediator release-associated symptoms could be directly related to degranulation of $\mathrm{MC}$, elicited by gas- 
Table 1. Characteristics of the anesthetic procedures and the cases that presented perianesthetic MC mediator release-related symptoms

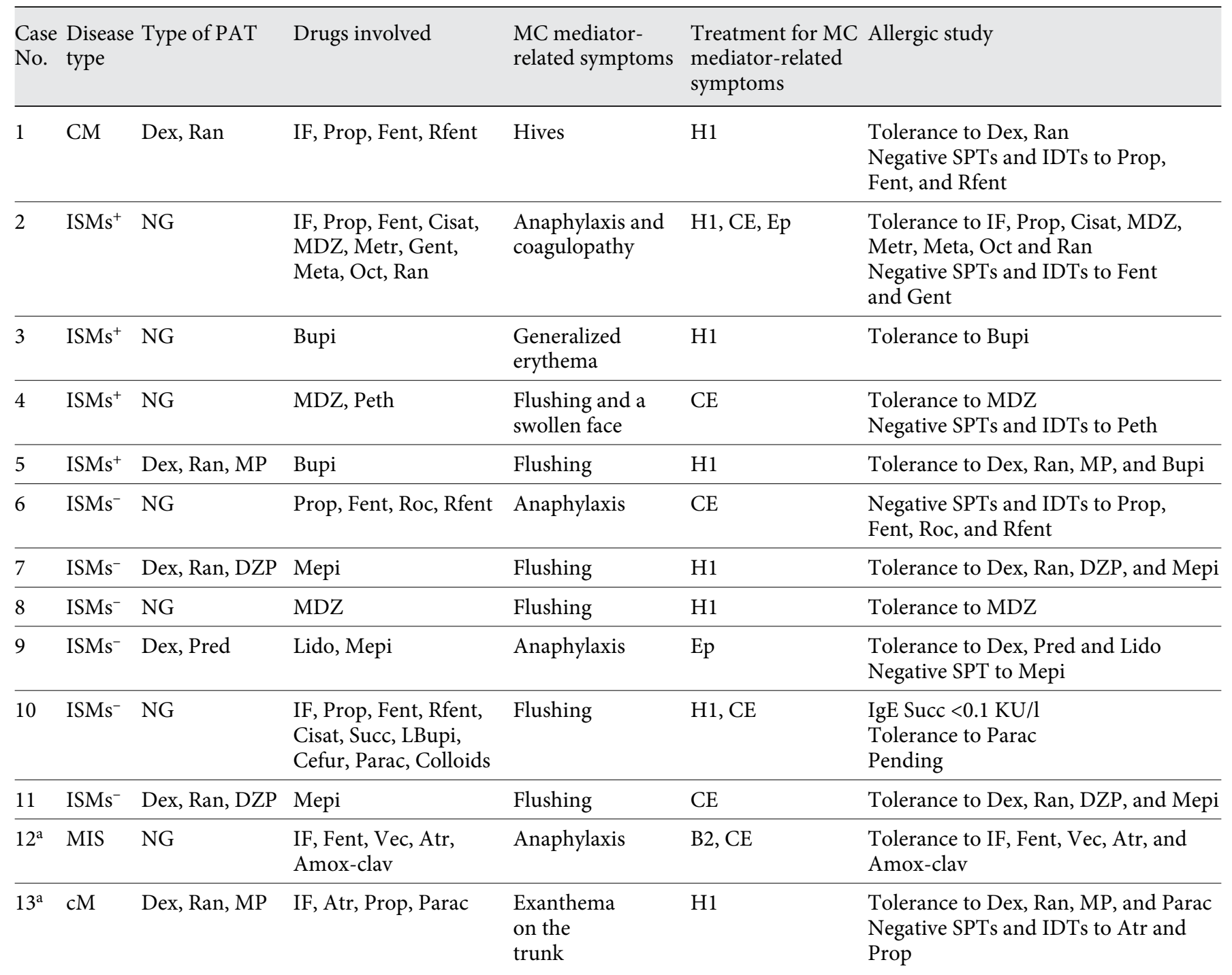

Amox-clav = Amoxicillin clavulanate; Atr = atropine; $\mathrm{B} 2=$ inhaled short-acting $\beta_{2}$-agonist Bupi $=$ bupivacaine; $\mathrm{CE}=$ corticosteroids; Cefur = cefuroxime; Cisat $=$ cisatracurium; $\mathrm{cM}=$ cutaneous mastocytoma; Dex $=$ dexchlorpheniramine; DZP $=$ diazepam; Ep $=$ epinephrine; Fent = fentanyl; Gent = gentamicin; $\mathrm{H} 1=\mathrm{H} 1$ antihistamines; $\mathrm{H} 2=\mathrm{H} 2$ antihistamines; $\mathrm{IF}=$ inhalants of the flurane family; IDT = intradermal skin test; LBupi = levobupivacaine; Lido = lidocaine; $\mathrm{MDZ}=$ midazolam; Mepi = mepivacaine; Meta = metamizole; Metr = metronidazole; $\mathrm{MP}=$ methylprednisolone; $\mathrm{NG}=$ not given; $\mathrm{Oct}=$ octeotride; Parac $=$ paracetamol; Peth = pethidine; Pred = prednisone; Prop = propofol; Ran = ranitidine; Rfent = remifentanil; Roc = rocuronium; SPT = skin prick test; Succ $=$ succinylcholine; Vec $=$ vecuronium. ${ }^{\text {a }}$ Pediatric case.

trointestinal manipulation (case No. 2) and pain and anxiety (No. 9) in 2 of 4 cases that presented anaphylaxis. Five (1\%) adult cases displayed increased bleeding and required a blood transfusion; 1 of these 5 cases (case No. 2) had an associated coagulopathy. In this study, none of the patients died due to MC mediator release induced by the anesthetic procedure.
The clinical data of adult and pediatric patients in the perioperative symptomatic group versus the asymptomatic group regarding demographics, categories of mastocytosis, symptoms, therapies, ASA classification, anesthetic techniques, and categories of surgery are shown in tables 2 and 3, respectively. Of note, no statistically significant differences were found among adult or pediatric 
Table 2. Clinical, biological, and treatment characteristics of adult cases $(n=676)$ grouped according to the presence or lack of perianesthetic MC mediator release-associated symptoms

\begin{tabular}{|c|c|c|c|}
\hline & $\begin{array}{l}\text { Asymptomatic } \\
(\mathrm{n}=665)\end{array}$ & $\begin{array}{l}\text { Symptomatic } \\
(\mathrm{n}=11)\end{array}$ & $\mathrm{p}$ value \\
\hline Female & $380(57)$ & $6(54)$ & NS \\
\hline \multicolumn{4}{|l|}{ Type of anesthesia } \\
\hline General & $62(9)$ & $4(36)$ & 0.02 \\
\hline Sedation & $65(10)$ & $2(18)$ & NS \\
\hline Epidural & $74(11)$ & $2(18)$ & NS \\
\hline Local & $511(77)$ & $4(36)$ & 0.005 \\
\hline Major surgery & $60(9)$ & $5(45)$ & $<0.001$ \\
\hline PAT given $1 \mathrm{~h}$ before anesthesia & $578(87)$ & $5(45)$ & 0.002 \\
\hline $\mathrm{H} 1$ antihistamines & $567(85)$ & $4(36)$ & $<0.001$ \\
\hline $\mathrm{H} 2$ antihistamines & $566(85)$ & $4(36)$ & $<0.001$ \\
\hline Corticosteroids & $67(10)$ & $2(18)$ & NS \\
\hline Montelukast & $16(2)$ & $0(0)$ & NS \\
\hline Benzodiacepines & $494(74)$ & $3(27)$ & 0.002 \\
\hline Median age at the anesthetic procedure, years & $45(17-78)$ & $41(17-65)$ & NS \\
\hline Median time from disease onset to anesthesia, months & $128(1-808)$ & $60(0-240)$ & NS \\
\hline Systemic mastocytosis & $624(94)$ & $10(91)$ & NS \\
\hline Nonaggressive mastocytosis & $599(87)^{\mathrm{a}}$ & $11(100)^{\mathrm{b}}$ & NS \\
\hline $\mathrm{BM} \mathrm{MC}^{\mathrm{c}}$ & $0.09(0-35)$ & $0.07(0.001-1)$ & NS \\
\hline sBT within 12 months prior to anesthesia, $\mathrm{ng} / \mathrm{ml}$ & $32.3(2-2,403)$ & $30.6(11.6-238)$ & NS \\
\hline \multicolumn{4}{|l|}{ Previous symptoms } \\
\hline Pruritus & $280(42)$ & $5(45)$ & NS \\
\hline Flushing & $238(36)$ & $7(64)$ & NS \\
\hline Abdominal cramping & $138(21)$ & $6(54)$ & NS \\
\hline Diarrhea & $154(23)$ & $5(45)$ & NS \\
\hline Anaphylaxis & $258(39)$ & $8(72)$ & 0.03 \\
\hline Anaphylaxis the year before & $96(14)$ & $5(45)$ & 0.02 \\
\hline \multicolumn{4}{|l|}{ Triggers of anaphylaxis before anesthesia } \\
\hline Idiopathic & $87(13)$ & $4(36)$ & 0.048 \\
\hline Insect sting & $116(17)$ & $3(27)$ & NS \\
\hline Drugs & $86(13)$ & $4(36)$ & 0.046 \\
\hline Foods & $19(3)$ & $0(0)$ & NS \\
\hline Anisakis simplex & $12(2)$ & $0(0)$ & NS \\
\hline Physical stimuli & $5(1)^{\mathrm{d}}$ & $0(0)$ & NS \\
\hline Scheduled antimediator therapy & $396(59)$ & $5(45)$ & NS \\
\hline H1 antihistamines & $52(8)$ & $0(0)$ & NS \\
\hline Disodium cromolyn & $164(25)$ & $2(18)$ & NS \\
\hline Intensive antimediator therapy & $180(27)$ & $3(27)$ & NS \\
\hline Epineprhine & $27(4)$ & $0(0)$ & NS \\
\hline Cytorreductive or targeted therapies ${ }^{\mathrm{e}}$ & $84(13)$ & $0(0)$ & NS \\
\hline Hydroxiurea & $17(2)$ & $0(0)$ & NS \\
\hline Interferon- $a-2 b$ & $20(3)$ & $0(0)$ & NS \\
\hline Pegylated interferon & $15(2)$ & $0(0)$ & NS \\
\hline Cladribine & $21(3)$ & $0(0)$ & NS \\
\hline Imatinib & $24(4)$ & $0(0)$ & NS \\
\hline Midostaurin & $1(0.1)$ & $0(0)$ & NS \\
\hline \multicolumn{4}{|l|}{ ASA score } \\
\hline I & $22(3)$ & $3(27)$ & 0.006 \\
\hline II & $541(81)$ & $8(73)$ & NS \\
\hline III & $100(15)$ & $0(0)$ & NS \\
\hline IV & $2(0.3)$ & $0(0)$ & NS \\
\hline
\end{tabular}

Values are expressed as numbers (\%) or medians (range). ISM-AHNMD = Indolent systemic mastocytosis associated with another clonal hematological non-mast cell lineage disease; $N S=$ not statistically significant $(\mathrm{p}>0.05) .{ }^{\mathrm{a}} \mathrm{CM}, \mathrm{n}=21 ; \mathrm{MIS}, \mathrm{n}=14$; $\mathrm{ISMs}^{+}, \mathrm{n}=355 ; \mathrm{ISMs}^{-}, \mathrm{n}=146 ;$ WDSM, $\mathrm{n}=57$, and ISM-AHNMD, $\mathrm{n}=6 .{ }^{\mathrm{b}} \mathrm{CM}, \mathrm{n}=1 ; \mathrm{ISMs}^{+}, \mathrm{n}=4 ; \mathrm{ISMs}^{-}, \mathrm{n}=6$. $^{\mathrm{c}}$ As assessed by flow cytometry. ${ }^{\mathrm{d}}$ Friction of skin mastocytoma. ${ }^{\mathrm{e}}$ Given to 44 adult patients who underwent 84 anesthetic procedures. 
Table 3. Clinical, biological, and treatment characteristics of the pediatric cases $(n=50)$ grouped according to the presence or absence of perianesthetic MC mediator release-associated symptoms

\begin{tabular}{|c|c|c|}
\hline & $\begin{array}{l}\text { Asymptomatic } \\
(\mathrm{n}=48)\end{array}$ & $\begin{array}{l}\text { Symptomatic } \\
(\mathrm{n}=2)\end{array}$ \\
\hline Female & $25(52)$ & $0(0)$ \\
\hline PAT given $1 \mathrm{~h}$ before anesthesia & $35(73)$ & $1(50)$ \\
\hline H1 antihistamines & $34(71)$ & $1(50)$ \\
\hline $\mathrm{H} 2$ antihistamines & $13(27)$ & $1(50)$ \\
\hline Corticosteorids & $9(19)$ & $1(50)$ \\
\hline Montelukast & $3(6)$ & $0(0)$ \\
\hline Benzodiacepines & $5(10)$ & $0(0)$ \\
\hline \multicolumn{3}{|l|}{ Anesthesia } \\
\hline General & $22(46)$ & $2(100)$ \\
\hline Sedation & $8(17)$ & $0(0)$ \\
\hline Epidural & $2(4)$ & $0(0)$ \\
\hline Local & $25(52)$ & $0(0)$ \\
\hline Major surgery & $11(23)$ & $2(100)$ \\
\hline Median age at the anesthetic procedure, months & $101(0-192)$ & $68(28-108)$ \\
\hline Median time from onset to anesthesia, months & $82(0-186)$ & $67(29-106)$ \\
\hline Extensive skin involvement & $2(4)$ & $0(0)$ \\
\hline Systemic mastocytosis & $3(6)$ & $0(0)$ \\
\hline sBT within 12 months previous to anesthesia, $\mathrm{ng} / \mathrm{ml}$ & $4.5(1.1-26.1)$ & $4.5(1.46-7.55)$ \\
\hline \multicolumn{3}{|l|}{ Previous symptoms } \\
\hline Pruritus & $16(33)$ & $0(0)$ \\
\hline Flushing & $13(27)$ & $0(0)$ \\
\hline Abdominal cramping & $10(21)$ & $0(0)$ \\
\hline Diarrhea & $8(17)$ & $0(0)$ \\
\hline Anaphylaxis & $6(12)$ & $0(0)$ \\
\hline \multicolumn{3}{|l|}{ Triggers of anaphylaxis before anesthesia } \\
\hline Idiopathic & $4(8)$ & $0(0)$ \\
\hline Drugs & $2(4)$ & $0(0)$ \\
\hline Scheduled antimediator therapy & $21(44)$ & $0(0)$ \\
\hline H1 antihistamines & $4(8)$ & $0(0)$ \\
\hline Disodium cromolyn & $6(12)$ & $0(0)$ \\
\hline Intensive antimediator therapy & $11(23)$ & $0(0)$ \\
\hline Epinephrine & $0(0)$ & $0(0)$ \\
\hline \multicolumn{3}{|l|}{ ASA score } \\
\hline I & $6(12)$ & $0(0)$ \\
\hline II & $40(83)$ & $0(0)$ \\
\hline III & $2(4)$ & $0(0)$ \\
\hline
\end{tabular}

Values are expressed as numbers (\%) or medians (range). No statistically significant differences $(\mathrm{p}>0.05)$ were found between the two groups. NA = No data available.

cases when they were grouped according to the presence or absence of systemic disease, the extent of skin involvement, and the percentage of BM MC and sBT levels during the year prior to the anesthetic procedure, and a higher ASA classification of cases was not associated with a higher frequency of MC mediator release-related symptoms (tables 2, 3). In contrast, the frequency of perioperative MC mediator release-associated symptoms was significantly higher in adult cases that underwent major surgery $(p<0.001)$ and general anesthesia $(p=0.02)$. In addition, adult cases showing perioperative MC mediator release-associated symptoms had more frequently had anaphylaxis $(\mathrm{p}=0.03)$, particularly idiopathic anaphylaxis (36 vs. $13 \%, \mathrm{p}<0.05)$ and anaphylaxis evoked by drugs ( 36 vs. $13 \%, p<0.05$ ), before the anesthesia in the perioperative symptomatic group despite the fact that known drug allergies were considered and those drugs were avoided in every individual case (tables 2, 3). Among the 
children, none of the 6 cases which had had previous anaphylactic episodes presented perioperative MC mediator release-related symptoms (table 3 ).

\section{Drugs Involved in the Anesthetic Procedures}

The drugs used as PAT (H1/H2 antihistamines, corticosteroids, montelukast, and benzodiacepines) were typically given $1 \mathrm{~h}$ before the procedure in the vast majority of both adult and pediatric cases; $\mathrm{H} 1$ antihistamines were the drugs more frequently used across the different types of anesthetic techniques; in turn, corticosteroids were preferentially used in general anesthesia among both adults (82\%) and children (75\%) (online suppl. table 2). Overall, the frequency of cases that did not get PAT among those that presented perioperative MC mediator release-associated symptoms was higher than that among cases that were given PAT (based on $\mathrm{H} 1 / \mathrm{H} 2$ antihistamines and benzodiacepines) $1 \mathrm{~h}$ before the anesthesia, i.e. 55 versus $13 \%$ of adults $(p=0.002)$ and 1 of 2 versus 13 of 48 children ( $p>0.05)$, respectively. The frequency of MC mediator release-associated symptoms did not show an association with the specific drugs administered for the anesthetic procedures in adults or in children (online suppl. table 3 ).

\section{Discussion}

Information about perioperative management and anesthesia in patients with mastocytosis in frequently based on case reports or small patient series [10, 12-14], which show low frequencies of moderate MC mediator release symptoms both in children (flushing, 9\%) [10] and in adults (7\%) [14], with a frequency of anaphylaxis of around $0.6 \%$ in adults [14]. In the present study, we report the largest series of use of anesthesia in adults and children with mastocytosis who have undergone anesthetic procedures. We found similar results in the pediatric and adult series, with a low frequency of moderate perioperative MC mediator release-associated symptoms (4 and 2\%, respectively); moreover, 1 child (2\%) and 3 adults $(0.3 \%)$ presented anaphylaxis, with the child even tolerating the same anesthetic procedure when PAT was used latter on. This frequency of perianesthetic MC mediator related symptoms is similar to that reported in the literature for children (although this series reports perianesthetic anaphylaxis, not previously described in other pediatric series), and it is lower than that previously described for adults, with a low frequency of anaphylaxis $(0.4 \%)$ similar to that found in the literature for mastocy-

Anesthesia in Patients with Mastocytosis tosis. Previous retrospective studies have reported an overall incidence of anaphylaxis (or anaphylactoid reactions) during anesthesia in the general population ranging from $1 / 3,500(0.03 \%)$ to $1 / 25,000(0.004 \%)$ [46-49]. Futhermore, prospective studies carried out in Spain [47, 50] have shown an incidence of perioperative anaphylaxis in the general population ranging from $1 / 10,263$ $(0.009 \%)$ to $1 / 7,085(0.01 \%)$, with the frequency being higher among subjects who have undergone general anesthesia $(1 / 6,973 ; 0.01 \%)$.

Altogether, these results support the notion that the frequency of perioperative anaphylaxis in patients with mastocytosis is higher than that observed in the general population. In addition, our results also indicate that anesthesia should not be avoided in patients with mastocytosis, particularly because the majority of cases have nonaggressive categories of the disease, with a good prognosis and a normal life expectancy. The frequency of perioperative anaphyalxis in our series was low, indicating that the risk for perioperative anaphylaxis in mastocytosis patients is most probably overestimated. Despite this, it is strongly recommended that anesthesia be managed as a high-risk procedure in these patients, since it seems to be difficult to predict and not directly related to the type of anesthesia used (e.g. general or local) or the presence of systemic disease as previously suggested $[12,32]$. Thus, perioperative MC mediator release-related symptoms were present in children with CM in the absence of extensive skin involvement or increased $\mathrm{sBT}$ (variables that are considered to be the major risk factors for severe $\mathrm{MC}$ mediator release-related symptoms and anaphylaxis in children with mastocytosis) [4, 51]. Similarly, MC degranulation-associated symptoms were frequently observed in adults with indolent categories of the disease independently of the overall MC burden as assessed by BM MC infiltration levels and sBT levels. Although the overall frequency of perioperative anaphylaxis was higher among the children than the adults, such a difference could be potentially due to the different number of procedures included in each series.

Overall, a relatively high heterogeneity was observed in our series regarding the administration of PAT before anesthetic procedures in mastocytosis patients, particularly for the anesthetic techniques performed outside the REMA centers, where the same protocol was followed in the majority of cases. Of note, such differences are probably related to the insufficient (empirical) data in the literature that would support the utility of PAT $[11,29,52$, $53]$, though some authors have proposed the use of premedication protocols in mastocytosis $[26,30,54]$, as well

Int Arch Allergy Immunol 2015;167:47-56 DOI: $10.1159 / 000436969$ 
as in other patients at risk of anaphylaxis, based on, for example, data on the medical history, previous asthma, and urticaria [55]. In this study, we found that PAT given $1 \mathrm{~h}$ before the anesthesia was not associated with any adverse effect, whereas it decreased the probability of suffering perioperative $\mathrm{MC}$ mediator release-associated symptoms, particularly when the PAT included $\mathrm{H} 1 / \mathrm{H} 2$ antihistamines and benzodiacepines. Furthermore, we observed that 3 of 13 symptomatic cases which underwent another anesthetic procedure tolerated the same anesthetic drugs when PAT was given $1 \mathrm{~h}$ before or implemented with adequate sedation. These results support the notion that stress and anxiety play an important role as elicitors of perioperative MC-related symptoms, as has been previously suggested $[8,11]$.

A complete allergic work-up is mandatory in mastocytosis patients who present perioperative MC mediator-related symptoms; accordingly, we recommend determining the specific IgE for the possible triggers (if available) and skin testing. If these results are negative and the drug involved has a low profile as elicitor of MC degranulation, a drug challenge under strict vigilance could be recommended. On the contrary, if the drug involved is highly suspicious of evoking MC degranulation (e.g. opioids and neuromuscular blockers), performing a challenge with PAT and other drugs of the pharmacological group that results in negative skin tests and a lower probability of eliciting MC degranulation could be a reasonable approach. Furthermore, we recommend increasing the PAT schedule if the previous one was not effective to prevent an episode of MC mediator release symptoms.

In this study, a remarkable number of perioperative symptomatic cases later tolerated the drugs involved in the $\mathrm{MC}$ degranulation episode, ruling them out as elicitors. However, only negative skin tests for the anesthetic drugs involved were found in the remaining cases; despite the great utility of these tests, they are not as good as a known later tolerance to the drug when ruling it out as the trigger (IgE mediated or not) of symptoms.

We cannot completely rule out that any of the drugs administered would have a greater risk of eliciting an episode of MC mediator release compared to other available drugs. Nevertheless, whenever possible, cautious patient management should be adopted in which drugs known to elicit MC degranulation are avoided and drugs known to be tolerated by individual patients are preferred [11, 13, $32,53,56,57]$; furthermore, if this information is not available or any of these drugs are indispensable, we recommend using the drug that probably has a lower risk of

eliciting MC degranulation (IgE mediated or not) in every pharmacological group, such as rigid neuromuscular blockers, synthetic opioids with a short-acting and rapid offset, and amide derivatives. Unfortunately, we could not estimate the predictive value of sBT determinations performed in the perioperative period (just before and/or after anesthesia), since it was not systematically assessed. Despite this, sBT levels determined in the year before the patient underwent anesthesia did not appear to be a reliable predictor of the occurrence of perioperative MC mediator release episodes.

In summary, based on the retrospective analysis of large series of adults and children with mastocytosis, we conclude that the frequency of MC perioperative symptoms in mastocytosis seems to be higher than that of the general population in the absence of a dramatic and fatal clinical outcome. Thus, mastocytosis is not a contraindication for the use of anesthesia, although it must be managed as a high-risk procedure in every patient and for every anesthetic technique, since risk factors for predicting the emergence of MC mediator release symptoms or anaphylaxis could not be identified. Despite this, PAT based on $\mathrm{H} 1 / \mathrm{H} 2$ antihistamines and/or benzodiacepines given $1 \mathrm{~h}$ before the anesthesia to avoid the stress is strongly recommended, as it was associated with fewer MC activation episodes in the absence of any adverse events related to this therapy.

\section{Acknowledgement}

This work was supported by grants from the Instituto de Salud Carlos III of the Ministerio de Economía y Competitividad of Spain (PS09-FEDER/00032, PI11/02399-FEDER, and RD12/0036/0048-RTICC), the Fundación Sociosanitaria de Castilla-La Mancha (FISCAM 2010/008 and G-2010/C-002), the Junta de Castilla y León (SAN/103/2011), the Fundación Ramón Areces, the Asociación Española de Pacientes de Mastocitosis (AEDM 2014), and the Hospital Virgen de la Salud Biobank (BioB-HVS) supported by grant of RETICS RD09/0076/00074 (Toledo, Spain).

\section{References}

Int Arch Allergy Immunol 2015;167:47-56
1 Escribano L, Alvarez-Twose I, Sanchez-Munoz L, Garcia-Montero A, Nunez R, Almeida $\mathrm{J}$, et al: Prognosis in adult indolent systemic mastocytosis: a long-term study of the Spanish Network on Mastocytosis in a series of 145 patients. J Allergy Clin Immunol 2009;124: 514-521.

2 Matito A, Morgado JM, Alvarez-Twose I, Sanchez-Muñoz L, Pedreira CE, Jara-Acevedo M, Teodosio C, et al: Serum tryptase monitoring in indolent systemic mastocytosis: association with disease features and patient outcome. PLoS One 2013;8:76116e. DOI: $10.1159 / 000436969$ 
3 Gonzalez de Olano D, de la Hoz B, NunezLopez R, Sanchez-Munoz L, Cuevas M, Dieguez C, et al: Prevalence of allergy and anaphylactic symptoms in 210 adult and pediatric patients with mastocytosis in Spain: a study of the Spanish network on mastocytosis (REMA). Clin Exp Allergy 2007;37:15471555.

4 Brockow K, Jofer C, Behrendt H, Ring J: Anaphylaxis in patients with mastocytosis: a study on history, clinical features and risk factors in 120 patients. Allergy 2008;63:226232.

5 Marone G, Stellato C: Activation of human mast cells and basophils by general anaesthetic drugs. Monogr Allergy 1992;30:5473.

6 Stellato C, Marone G: Mast cells and basophils in adverse reactions to drugs used during general anesthesia. Chem Immunol 1995;62:108131.

7 Alvarez-Twose I, Morgado JM, Sanchez-Munoz L, Garcia-Montero A, Mollejo M, Orfao $\mathrm{A}$, et al: Current state of biology and diagnosis of clonal mast cell diseases in adults. Int J Lab Hematol 2012;34:446-460.

8 Escribano L, Akin C, Castells M, Orfao A, Metcalfe D: Mastocytosis: current concepts in diagnosis and treatment. Ann Hematol 2002; 81:677-690.

9 Stellato C, De Paulis A, Cirillo R, Mastronardi P, Mazzarella B, Marone G: Heterogeneity of human mast cells and basophils in response to muscle relaxants. Anesthesiology 1991;74: 1078-1086.

10 Carter MC, Uzzaman A, Scott LM, Metcalfe DD, Quezado Z: Pediatric mastocytosis: routine anesthetic management for a complex disease. Anesth Analg 2008;107:422-427.

11 Dewachter P, Castells MC, Hepner DL, Mouton-Faivre C: Perioperative management of patients with mastocytosis. Anesthesiology 2014;120:753-759.

12 James PD, Krafchik BR, Johnston AE: Cutaneous mastocytosis in children: anaesthetic considerations. Can J Anaesth 1987;34:522524.

13 Ahmad N, Evans P, Lloyd-Thomas AR: Anesthesia in children with mastocytosis - a case based review. Paediatr Anaesth 2009;19:97107.

14 Matito A, Alvarez-Twose I, Sanchez-Munoz L, Manzanero M, Escribano L: Frequency of mast cells-mediator related symptoms during anesthesia in patients with mastocytosis: a retrospective study in 148 risk procedures with anesthesia by the Spanish Network on Mastocytosis (REMA). Allergy 2010;65:S345S346.

15 Coleman MA, Liberthson RR, Crone RK, Levine FH: General anesthesia in a child with urticaria pigmentosa. Anesth Analg 1980;59: 704-706.

16 Scott HW Jr, Parris WC, Sandidge PC, Oates JA, Roberts LJ: Hazards in operative management of patients with systemic mastocytosis. Ann Surg 1983;197:507-514.
17 Hosking MP, Warner MA: Sudden intraoperative hypotension in a patient with asymptomatic urticaria pigmentosa. Anesth Analg 1987;66:344-346.

18 Smith GB, Gusberg RJ, Jordan RH, Kim B: Histamine levels and cardiovascular responses during splenectomy and splenorenal shunt formation in a patient with systemic mastocytosis. Anaesthesia 1987;42: 861-867.

19 Desborough JP, Taylor I, Hattersley A, Garden A, Wolff A, Bloom SR, et al: Massive histamine release in a patient with systemic mastocytosis. Br J Anaesth 1990;65:833836.

20 Greenblatt EP, Chen L: Urticaria pigmentosa: an anesthetic challenge. J Clin Anesth 1990;2: 108-115.

21 Ojeda A, Crespo A, Crespo V, Sánchez F, Sanz A, Vera A: Telangiectasia maculosa eruptiva persistente con afectación sistémica y evolución postoperatoria fatal. Actas Dermosifiliogr 1996;87:539-542.

22 Vaughan STA, Jones GN: Systemic mastocytosis presenting as profound cardiovascular collapse during anaesthesia. Anaesthesia 1998;53:804-807.

23 Tirel O, Chaumont A, Ecoffey C: Circulatory arrest in the course of anesthesia for a child with mastocytosis. Ann Fr Anesth Reanim 2001;20:874-875.

24 Brodier C, Guyot E, Palot M, David P, Rendoing J: Anesthesia of a child with a cutaneous mastocytosis. Cah Anesthesiol 1993;41:7779.

25 Bridgman DE, Clarke R, Sadleir PH, Stedmon JJ, Platt P: Systemic mastocytosis presenting as intraoperative anaphylaxis with atypical features: a report of two cases. Anaesth Intensive Care 2013;41:116-121.

26 Lerno G, Slaats G, Coenen E, Herregods L, Rolly G: Anaesthetic management of systemic mastocytosis. Br J Anaesth 1990;65:254257.

27 Yaniv R, Segal E, Perel A: Anesthetic considerations in mast-cell proliferative disease (urticaria pigmentosa and mastocytosis). Harefuah 1992;122:780-784.

28 Chaar CI, Bell RL, Duffy TP, Duffy AJ: Guidelines for safe surgery in patients with systemic mastocytosis. Am Surg 2009;75:74-80.

29 Dewachter P, Mouton-Faivre C, Cazalaa JB, Carli P, Lortholary O, Hermine O: Mastocytosis and anaesthesia. Ann Fr Anesth Reanim 2009;28:61-73.

30 Konrad FM, Schroeder TH: Anaesthesia in patients with mastocytosis. Acta Anaesthesiol Scand 2009;53:270-271.

31 Calvo N, Telletxea S, Intxaurraga K, Arizaga A: Systemic mastocytosis and perioperative management: a report of 2 cases. Rev Esp Anestesiol Reanim 2010;57:192-194.

32 Brockow K, Bonadonna P: Drug allergy in mast cell disease. Curr Opin Allergy Clin Immunol 2012;12:354-360

33 Delalande JP, Rea D, Fenoll B: Absence of mast cell degranulation during general anes- thesia in a child with mastocytosis. Ann Fr Anesth Reanim 1992;11:393-394.

34 Koitabashi T, Takino Y: Anesthetic management of a patient with urticaria pigmentosa. Masui 1995;44:279-281.

35 Borgeat A, Ruetsch YA: Anesthesia in a patient with malignant systemic mastocytosis using a total intravenous anesthetic technique. Anesth Analg 1998;86:442-444.

36 Auvray L, Letourneau B, Freysz M: Mastocytosis: general anesthesia with remifentanil and sevoflurane. Ann Fr Anesth Reanim 2001;20:635-638.

37 Fisher MM, Baldo BA: Mast cell tryptase in anaesthetic anaphylactoid reactions. Br J Anaesth 1998;80:26-29.

38 Lorenzi P, Filoni M, Manetta G, Bonechi ML, Salvati G, Tanini A: Anaphylactic reaction to thiopental: a case documented by tryptase values and RAST. Minerva Anestesiol 1999;65: 659-663.

39 Valent P, Horny HP, Li CY, Longley BJ, Metcalfe DD, Parwaresch MR, et al: Mastocytosis (mast cell disease); in Jaffe ES, Harris NL, Stein H, Vardiman JW (eds): WHO Classification of Tumours: Pathology and Genetics - Tumours of Haematopoietic and Lymphoid Tissues. Lyon, IARC Press, 2001, vol 1, pp 291-302.

40 Valent P, Horny HP, Escribano L, Longley-BJ J, Li CL, Schwartz LB, et al: Diagnostic criteria and classification of mastocytosis: a consensus proposal. Leuk Res 2001;25:603-625.

41 Valent P, Akin C, Escribano L, Fodinger M, Hartmann K, Brockow K, et al: Standards and standardization in mastocytosis: consensus statements on diagnostics, treatment recommendations and response criteria. Eur J Clin Invest 2007;37:435-453.

42 Horny HP, Metcalfe DD, Bennet JM, Bain BJ, Akin C, Escribano L, et al: Mastocytosis; in Swerdlow SH, Campo E, Harris NL, Jaffe ES, Pileri SA, Stein H, Thiele J, Vardiman JW (eds): WHO Classification of Tumours of Haematopoietic and Lymphoid Tissues. Lyon, IARC, 2008, pp 54-63.

43 Akin C, Fumo G, Yavuz AS, Lipsky PE, Neckers L, Metcalfe DD: A novel form of mastocytosis associated with a transmembrane c-kit mutation and response to imatinib. Blood 2004; 103:3222-3225.

44 ASA Relative Value Guide. 2013. www.asaqh. org.

45 Earl R: Definition of major and minor surgery: a question and an answer. Ann Surg 1917;65:799.

46 Currie M, Webb RK, Williamson JA, Russell WJ, Mackay P: The Australian Incident Monitoring Study: clinical anaphylaxis - an analysis of 2,000 incident reports. Anaesth Intensive Care 1993;21:621-625.

47 Escolano F, Valero A, Huguet J, Baxarias P, de Molina M, Castro A, et al: Prospective epidemiologic study of perioperative anaphylactoid reactions occurring in Catalonia (1996-7). Rev Esp Anestesiol Reanim 2002, 49:286-293. 
48 Kroigaard M, Garvey LH, Gillberg L, Johansson SG, Mosbech H, Florvaag E, et al: Scandinavian Clinical Practice Guidelines on the diagnosis, management and follow-up of anaphylaxis during anaesthesia. Acta Anaesthesiol Scand 2007;51:655670.

49 Lieberman P, Nicklas RA, Oppenheimer J, Kemp SF, Lang DM, Bernstein DI, et al: The diagnosis and management of anaphylaxis practice parameter: 2010 update. J Allergy Clin Immunol 2010;126:477-480.

50 Navarro J, Escolano F, Sánchez JC, Mansilla $\mathrm{R}$, Castillo J: Incidence of severe perioperative anaphylactoid reactions. Eur J Anaesthesiol 2003;23:A-26.
51 Alvarez-Twose I, Vano-Galvan S, SanchezMunoz L, Morgado JM, Matito A, Torrelo A, et al: Increased serum baseline tryptase levels and extensive skin involvement are predictors for the severity of mast cell activation episodes in children with mastocytosis. Allergy 2012; 67:813-821.

52 Bonadonna P, Lombardo C, Zanotti R: Mastocytosis and allergic diseases. J Investig Allergol Clin Immunol 2014;24:288-297.

53 Bonadonna P, Lombardo C: Drug allergy in mastocytosis. Immunol Allergy Clin North Am 2014;34:397-405.

54 Matito A, Alvarez-Twose I, Morgado JM, Sanchez-Munoz L, Orfao A, Escribano L: Clinical impact of pregnancy in mastocytosis: a study of the Spanish Network on Mastocytosis (REMA) in 45 cases. Int Arch Allergy Immunol 2011;156:104-111.

55 Manfredi G, Pezzuto F, Balestrieri A, Lo SM, Montera MC, Pio A, et al: Perioperative anaphylactic risk score for risk-oriented premedication. Transl Med UniSa 2013;7:12-17.

56 Mertes PM, Malinovsky JM, Jouffroy L, Aberer W, Terreehorst I, Brockow K, et al: Reducing the risk of anaphylaxis during anesthesia: 2011 updated guidelines for clinical practice. J Investig Allergol Clin Immunol 2011; 21:442-453.

57 Klein NJ, Misseldine S: Anesthetic considerations in pediatric mastocytosis: a review. J Anesth 2013;27:588-598. 